ESAIM: COCV 19 (2013) 1055-1063

DOI: $10.1051 / \mathrm{cocv} / 2012044$
ESAIM: Control, Optimisation and Calculus of Variations

www.esaim-cocv.org

\title{
NOTE ON THE INTERNAL STABILIZATION OF STOCHASTIC PARABOLIC EQUATIONS WITH LINEARLY MULTIPLICATIVE GAUSSIAN NOISE*
}

\author{
VIOREL BARBU ${ }^{1}$
}

\begin{abstract}
The parabolic equations driven by linearly multiplicative Gaussian noise are stabilizable in probability by linear feedback controllers with support in a suitably chosen open subset of the domain. This procedure extends to Navier-Stokes equations with multiplicative noise. The exact controllability is also discussed.
\end{abstract}

Mathematics Subject Classification. 35Q30, 60H15, 35B40.

Received April 26, 2012. Revised November 3, 2012.

Published online July 4, 2013.

\section{INTRODUCTION}

Consider the stochastic nonlinear controlled parabolic equation

$$
\begin{aligned}
& \mathrm{d} X(t)-\Delta X(t) \mathrm{d} t+a(t, \xi) X(t) \mathrm{d} t+b(t, \xi) \cdot \nabla_{\xi} X(t) \mathrm{d} t \\
& \quad+f(X(t)) \mathrm{d} t=X(t) \mathrm{d} W(t)+\mathbf{1}_{\mathcal{O}_{0}} u(t) \mathrm{d} t \quad \text { in }(0, \infty) \times \mathcal{O}, \\
& X=0 \quad \text { on }(0, \infty) \times \partial \mathcal{O}, \quad X(0)=x \quad \text { in } \mathcal{O} .
\end{aligned}
$$

Here, $\mathcal{O}$ is a bounded and open domain of $\mathbb{R}^{d}, d \geq 1$, with smooth boundary $\partial \mathcal{O}$ and $W(t)$ is a Wiener process of the form

$$
W(t)=\sum_{k=1}^{\infty} \mu_{k} e_{k}(\xi) \beta_{k}(t), \quad t \geq 0, \xi \in \mathcal{O},
$$

where $\mu_{k}$ are real numbers, $\left\{e_{k}\right\}$ is an orthonormal basis in $L^{2}(\mathcal{O}),\left\{e_{k}\right\} \subset C^{2}(\overline{\mathcal{O}})$ and $\left\{\beta_{k}\right\}_{k=1}^{\infty}$ are independent Brownian motions in a stochastic basis $\left\{\Omega, \mathcal{F}, \mathcal{F}_{t}, \mathbb{P}\right\}$. Throughout this work, we assume that

$$
\sum_{k=1}^{\infty} \mu_{k}^{2}\left|e_{k}\right|_{\infty}^{2}<\infty
$$

where $|\cdot|_{\infty}$ denotes the $L^{\infty}(\mathcal{O})$-norm.

\footnotetext{
Keywords and phrases. Stochastic equation, brownian motion, Navier-Stokes equation, feedback controller.

* Supported by a grant of the Romanian National Authority for Scientific Research, CNCS-UEFISCDI Project PN-II-ID-PCE2011-3-0027.

1 Al.I. Cuza University and Octav Mayer Institute of Mathematics (Romanian Academy), Blvd. Carol I, No. 11, 700506 Iaşi, Romania. viorelbarbu<vbarbu41@gmail.com>
} 
The function $a:[0, \infty) \times \mathcal{O} \rightarrow \mathbb{R}, b:[0, \infty) \times \mathcal{O} \rightarrow \mathbb{R}^{d}$ and $f: \mathbb{R} \rightarrow \mathbb{R}$ are assumed to satisfy

$$
\begin{gathered}
a \in C([0, \infty) \times \overline{\mathcal{O}}), b \in C^{1}([0, \infty) \times \overline{\mathcal{O}}) \\
\sup \left\{|a(t)|_{\infty}+|\nabla b(t)|_{\infty} ; t \geq 0\right\}<\infty \\
f \in \operatorname{Lip}(\mathbb{R}), f(0)=0 .
\end{gathered}
$$

Finally, $\mathcal{O}_{0}$ is an open subdomain of $\mathcal{O}$ with smooth boundary, $\mathbb{1}_{\mathcal{O}_{0}}$ is its characteristic function and $u=u(t, \xi)$ is an adapted controller with respect to the natural filtration $\left\{\mathcal{F}_{t}\right\}$.

The main problem we address here is the design of a feedback controller $u=F(X)$ such that the corresponding closed loop system (1.1) is asymptotically stable in probability, that is,

$$
\lim _{t \rightarrow \infty} X(t)=0 \quad \text { in } \quad L^{2}(\mathcal{O}), \mathbb{P} \text {-a.s. }
$$

The construction of this stabilizing feedback controller is given in Theorem 2.1 and this idea was already used to stabilize the deterministic Navier-Stokes equation [4]. However, the extention to the stochastic case is not immediate and less obvious; some sharper arguments involving the martingale convergence theorem are necessary. It should be said, in this context, that a stronger property, the exact controllability of (1.1) in finite time, is in general still an open problem. (See, however, $[5,9,11,14]$ for partial results). A similar result is established (see Thm. 4.1) for the $2-D$ Navier-Stokes equations with multiplicative noise.

\section{Notation}

By $L^{2}(\mathcal{O})$ we denote the space of all Lebesgue square integrable functions on $\mathcal{O}$ with the norm $|\cdot|_{2}$ and the scalar product $\langle\cdot, \cdot\rangle$. The scalar product of $L^{2}\left(\mathcal{O}_{0}\right)$ is denoted by $\langle\cdot, \cdot\rangle_{0}$. If $Y$ is a Banach space with the norm $\|\cdot\|_{Y}$, we denote by $L^{p}(0, T ; Y), 1 \leq p \leq \infty$, the space of all Bochner measurable functions $u:(0, T) \rightarrow Y$ with $\|u\|_{Y} \in L^{p}(0, T)$. By $C([0, T] ; Y)$, we denote the space of all continuous $Y$-valued functions on $[0, T]$. We denote also by $H^{k}(\mathcal{O}), k=1,2$, the standard Sobolev space of functions on $\mathcal{O}, H_{0}^{1}(\mathcal{O})=\left\{y \in H^{1}(\mathcal{O}) ; y=0\right.$ on $\left.\partial \mathcal{O}\right\}$.

Given an $\mathcal{F}_{t}$-adapted process $u \in L^{2}\left(0, T ; L^{2}\left(\Omega, L^{2}(\mathcal{O})\right)\right)$, an $\mathcal{F}_{t^{-}}$adapted process $X:[0, T] \rightarrow L^{2}(\mathcal{O})$ with $\mathbb{P}$-a.s. continuous sample paths is said to be a solution to $(1.1)$ if it is in $C\left([0, T] ; L^{2}\left(\Omega ; L^{2}(\mathcal{O})\right)\right)$ and

$$
\begin{array}{r}
X(t, \xi)=x+\int_{0}^{t}\left(\Delta X(s, \xi)-a(t, \xi) X(s, \xi)-b(s, \xi) \cdot \nabla_{\xi} X(s, \xi)\right. \\
+f(X(s, \xi))) \mathrm{d} s+\int_{0}^{t} \mathbf{1}_{\mathcal{O}_{0}} u(s, \xi) \mathrm{d} s+\int_{0}^{t} X(s, \xi) \mathrm{d} W(s), \\
\xi \in \mathcal{O}, t \in(0, T), \mathbb{P} \text {-a.s. },
\end{array}
$$

where the integral is considered in sense of Itô with respect to the Wiener process $W$ (see [10]). We refer to [10], Proposition 6.1, for the existence and uniqueness of such a solution. Moreover, by the Burkholder-Davis-Gundy theorem, if follows also that $X \in L^{2}\left(\Omega ; L^{\infty}\left(0, T ; L^{2}(\mathcal{O})\right)\right)$.

\section{The Stabilization of EQUation (1.1)}

We set $\mathcal{O}_{1}=\mathcal{O} \backslash \overline{\mathcal{O}}_{0}$ and denote by $A_{1}: D\left(A_{1}\right) \subset L^{2}\left(\mathcal{O}_{1}\right) \rightarrow L^{2}(\mathcal{O})$ defined by

$$
A_{1} y=-\Delta y, y \in D\left(A_{1}\right)=H_{0}^{1}\left(\mathcal{O}_{1}\right) \cap H^{2}\left(\mathcal{O}_{1}\right)
$$

or, equivalently,

$$
\left\langle A_{1} y, z\right\rangle_{1}=\int_{\mathcal{O}_{1}} \nabla y \cdot \nabla z \mathrm{~d} \xi, \forall y, z \in H_{0}^{1}\left(\mathcal{O}_{1}\right),
$$

where $\langle\cdot, \cdot\rangle_{1}$ is the duality on $H_{0}^{1}\left(\mathcal{O}_{1}\right) \times H^{-1}\left(\mathcal{O}_{1}\right)$ induced by $L^{2}\left(\mathcal{O}_{1}\right)$ as pivot space. 
Denote by $\lambda_{1}^{*}\left(\mathcal{O}_{1}\right)$ the first eigenvalue of the operator $A_{1}$, that is,

$$
\lambda_{1}^{*}\left(\mathcal{O}_{1}\right)=\inf \left\{\int_{\mathcal{O}_{1}}|\nabla y|^{2} \mathrm{~d} \xi ; y \in H_{0}^{1}\left(\mathcal{O}_{1}\right), \int_{\mathcal{O}_{1}} y^{2} \mathrm{~d} \xi=1\right\} .
$$

Consider in (1.1) the feedback controller

$$
u=-\eta X, \eta \in \mathbb{R}^{+}
$$

and the corresponding closed loop system

$$
\begin{aligned}
& \mathrm{d} X-\Delta X \mathrm{~d} t+a X \mathrm{~d} t+b \cdot \nabla X \mathrm{~d} t+f(X) \mathrm{d} t=X \mathrm{~d} t-\eta \mathbb{1}_{\mathcal{O}_{0}} X \mathrm{~d} t+X \mathrm{~d} W \text { in }(0, \infty) \times \mathcal{O}, \\
& X(0)=x \text { in } \mathcal{O} \\
& X=0 \text { on }(0, \infty) \times \partial \mathcal{O} .
\end{aligned}
$$

Theorem 2.1 is the main result.

Theorem 2.1. Assume that

$$
\lambda_{1}^{*}\left(\mathcal{O}_{1}\right)-\frac{1}{2} \sum_{j=1}^{\infty} \mu_{j}^{2}\left|e_{j}\right|_{\infty}^{2}-\|f\|_{\text {Lip }}-\sup \left\{-a(t, \xi)+\frac{1}{2} \operatorname{div}_{\xi} b(t, \xi) ;(t, \xi) \in \mathbb{R}^{+} \times \mathcal{O}\right\}>0 .
$$

Then, for each $x \in L^{2}(\mathcal{O})$ and for $\eta$ sufficiently large (independent of $x$ ), the feedback controller (2.10) exponentially stabilizes in probability equation (1.1). More precisely, there is a $\gamma>0$ such that the solution $X$ to (2.11) satisfies

$$
\begin{gathered}
\lim _{t \rightarrow \infty} \mathrm{e}^{\gamma t}|X(t)|_{2}^{2}=0, \mathbb{P} \text {-a.s. } \\
\mathrm{e}^{\gamma t} \mathbb{E}|X(t)|_{2}^{2}+\mathbb{E} \int_{0}^{\infty} \mathrm{e}^{\gamma t}|X(t)|_{2}^{2} \mathrm{~d} t \leq C|x|_{2}^{2} .
\end{gathered}
$$

We recall that, by the classical Rayleigh-Faber-Krahn perimetric inequality in dimension $d \geq 1$, we have

$$
\lambda_{1}^{*}\left(\mathcal{O}_{1}\right) \geq\left(\frac{\omega_{d}}{\left|\mathcal{O}_{1}\right|}\right)^{\frac{2}{d}} J_{\frac{d}{2}-1,1}
$$

where $\left|\mathcal{O}_{1}\right|=\operatorname{Vol}\left(\mathcal{O}_{1}\right), \omega_{d}=\pi^{\frac{d}{2}} /\left(\Gamma\left(\frac{d}{2}+1\right)\right)$, and $J_{m, 1}$ is the first positive zero of the Bessel function $I_{m}(r)$.

Hence, by Theorem 2.1, we conclude that, if $\left|\mathcal{O}_{1}\right|$ is sufficiently small, then the feedback controller $(2.10)$ exponentially stabilizes the system. More precisely, we have

Corollary 2.2. Assume that

$$
\left|\mathcal{O}_{1}\right|<\omega_{d} J_{\frac{d}{2}-1,1}^{\frac{d}{2}}\left(\frac{1}{2} \sum_{j=1}^{\infty} \mu_{j}^{2}\left|e_{j}\right|_{\infty}^{2}+\sup _{\mathbb{R}^{+} \times \mathcal{O}}\left\{-a+\frac{1}{2} \operatorname{div}_{\xi} b\right\}+\|f\|_{\text {Lip }}\right)^{-\frac{d}{2}}
$$

Then, for each $x \in L^{2}(\mathcal{O})$ and $\eta$ sufficiently large, the feedback controller (2.10) stabilizes system (1.1) in sense of (2.13), (2.14).

We note that, in particular, condition (2.16) is satisfied if the Hausdorff distance $d_{H}\left(\partial \mathcal{O}, \partial \mathcal{O}_{0}\right)$ is sufficiently small. 
Remark 2.3. One might suspect that system (1.1) is stabilizable and even exact null controllable in probability by controllers $u$ with support in an arbitrary open subset $\mathcal{O}_{0} \subset \mathcal{O}$, as is the case in the deterministic case (see, e.g., $[2,3,7]$ ), but so far this is an open problem. (More will be said about this in Sect. 5 below).

Roughly speaking, Theorem 2.1 amounts to saying that the stochastic perturbation destabilizing effect in system (1.1) can be compensated by a linear stabilizing feedback controller with support in a subdomain $\mathcal{O}_{0}$ satisfying (2.16).

An example. The stochastic equation

$$
\begin{aligned}
& \mathrm{d} X-X_{\xi \xi} \mathrm{d} t+\left(a X+b X_{\xi}\right) \mathrm{d} t=\mu X \mathrm{~d} \beta+V \mathrm{~d} t, 0<\xi<1, \\
& X(t, 0)=X(t, 1)=0, t \geq 0,
\end{aligned}
$$

where $\beta$ is a Brownian motion and $\mu \in \mathbb{R}, a \in C([0, T] \times \mathbb{R}), b \in C^{1}([0,1] \times \mathbb{R})$, is exponentially stabilizable in probability by any feedback controller $V=-\eta \mathbf{1}_{\left[a_{1}, a_{2}\right]} X$, where $\eta>0$ is sufficiently large and $0<a_{1}<a_{2}<1$ are such that (see (2.12))

$$
\left(\frac{\pi}{a_{2}-a_{1}}\right)^{2}>\frac{\mu^{2}}{2}+\sup _{(t, \xi) \in \mathbb{R}^{+} \times(0,1)}\left\{-a(t, \xi)+\frac{1}{2} b_{\xi}(t, \xi)\right\} .
$$

\section{Proof of Theorem 2.1}

The main ingredient of the proof is the following lemma.

Lemma 3.1. For each $\varepsilon>0$ there is $\eta_{0}=\eta_{0}(\varepsilon)$ such that

$$
\int_{\mathcal{O}}|\nabla y(\xi)|^{2} \mathrm{~d} \xi+\eta \int_{\mathcal{O}_{0}} y^{2}(\xi) \mathrm{d} \xi \geq\left(\lambda_{1}^{*}\left(\mathcal{O}_{1}\right)-\varepsilon\right)|y|_{2}^{2}, \forall y \in H_{0}^{1}(\mathcal{O}), \eta \geq \eta_{0} .
$$

The proof is well known (see $[1,4]$ ), but we outline it for the sake of completeness. Denote by $\nu_{1}^{\eta}$ the first eigenvalue of the self-adjoint operator

$$
A^{\eta} y=A y+\eta \mathbf{1}_{\mathcal{O}_{0}} y, \forall y \in D\left(A^{\eta}\right)=H_{0}^{1}(\mathcal{O}) \cap H^{2}(\mathcal{O}),
$$

where $A=-\Delta, D(A)=H_{0}^{1}(\mathcal{O}) \cap H^{2}(\mathcal{O})$ and $\eta \in \mathbb{R}^{+}$.

We have by the Rayleigh formula

$$
\nu_{1}^{\eta}=\inf \left\{\int_{\mathcal{O}}|\nabla y|^{2} \mathrm{~d} \xi+\eta \int_{\mathcal{O}_{0}}|y|^{2} \mathrm{~d} \xi ;|y|_{2}=1\right\} \leq \lambda_{1}^{*}\left(\mathcal{O}_{1}\right)
$$

because any function $y \in H_{0}^{1}\left(\mathcal{O}_{1}\right)$ can be extended by zero to $H_{0}^{1}(\mathcal{O})$ across the smooth boundary $\partial \mathcal{O}_{1}=\partial \mathcal{O}_{0}$. Let $\varphi_{1}^{\eta} \in H_{0}^{1}(\mathcal{O}) \cap H^{2}(\mathcal{O})$ be such that

$$
A^{\eta} \varphi_{1}^{\eta}=\nu_{1}^{\eta} \varphi_{1}^{\eta}, \quad\left|\varphi_{1}^{\eta}\right|_{2}=1
$$

We have by (3.18) that

$$
\int_{\mathcal{O}}\left|\nabla \varphi_{1}^{\eta}\right|^{2} \mathrm{~d} \xi+\eta \int_{\mathcal{O}_{0}}\left|\varphi_{1}^{\eta}\right|^{2} \mathrm{~d} \xi=\nu_{1}^{\eta} \leq \lambda_{1}^{*}(\mathcal{O}), \forall \eta>0
$$

Then, on a subsequence, again denoted $\eta$, we have for $\eta \rightarrow \infty$, that $\nu_{1}^{\eta} \rightarrow \nu^{*}$ and

$$
\begin{aligned}
\varphi_{1}^{\eta} & \rightarrow \varphi_{1} \text { weakly in } H_{0}^{1}(\mathcal{O}), \text { strongly in } L^{2}(\mathcal{O}) \\
\int_{\mathcal{O}_{0}}\left|\varphi_{1}^{\eta}\right|^{2} \mathrm{~d} \xi & \rightarrow 0 .
\end{aligned}
$$


We have, therefore, $\varphi_{1} \in H_{0}^{1}\left(\mathcal{O}_{1}\right),\left|\varphi_{1}\right|_{L^{2}\left(\mathcal{O}_{0}\right)}=0$ and $A_{1} \varphi_{1}=\nu^{*} \varphi_{1}$. Moreover, by $(3.18)$ we see that $\nu^{*} \leq \lambda_{1}^{*}\left(\mathcal{O}_{1}\right)$. Since $\lambda_{1}^{*}\left(\mathcal{O}_{1}\right)$ is the first eigenvalue of $A_{1}$, we have that $\nu^{*}=\lambda_{1}^{*}\left(\mathcal{O}_{1}\right)$ and so, by (3.18) we have

$$
\lim _{\eta \rightarrow \infty} \inf \left\{\int_{\mathcal{O}}|\nabla y|^{2} \mathrm{~d} \xi+\eta \int_{\mathcal{O}_{0}} y^{2} \mathrm{~d} \xi ;|y|_{2}=1\right\}=\lambda_{1}^{*}\left(\mathcal{O}_{1}\right)
$$

This yields (3.17), as claimed.

Proof of Theorem 2.1 (continued). By applying Itô's formula in (2.11) to the function $\varphi(t, x)=\frac{1}{2} \mathrm{e}^{\gamma t}|x|_{2}^{2}$, we obtain that

$$
\begin{aligned}
\frac{1}{2} \mathrm{~d}\left(\mathrm{e}^{\gamma t}|X(t)|_{2}^{2}\right)+\int_{\mathcal{O}} \mathrm{e}^{\gamma t}|\nabla X(t, \xi)|^{2} \mathrm{~d} \xi \\
\quad+\int_{\mathcal{O}} \mathrm{e}^{\gamma t}\left(a(t, \xi)-\frac{\gamma}{2}-\frac{1}{2} \operatorname{div}_{\xi} b(t, \xi) X^{2}(t, \xi)+f(X(t, \xi)) X(t, \xi)\right) \mathrm{d} \xi \\
=\frac{1}{2} \int_{\mathcal{O}} \mathrm{e}^{\gamma t} \sum_{k=1}^{\infty}\left|\left(X e_{k}\right)(t, \xi)\right|^{2} \mathrm{~d} \xi-\eta \int_{\mathcal{O}_{0}} \mathrm{e}^{\gamma t}|X(t, \xi)|^{2} \mathrm{~d} \xi \\
\quad+\int_{\mathcal{O}} \mathrm{e}^{\gamma t} \sum_{k=1}^{\infty}\left(X e_{k}\right)(t, \xi) X(t, \xi) \mathrm{d} \beta_{k}(t), \mathbb{P} \text {-a.s., } t \geq 0 .
\end{aligned}
$$

Equivalently,

$$
\frac{1}{2} \mathrm{e}^{\gamma t}|X(t)|_{2}^{2}+\int_{0}^{t} K(s) \mathrm{d} s=\frac{1}{2}|x|_{2}^{2}+M(t), t \geq 0, \mathbb{P} \text {-a.s. }
$$

where

$$
\begin{gathered}
K(t)=\int_{\mathcal{O}} \mathrm{e}^{\gamma t}\left(|\nabla X(t, \xi)|^{2}+\left(a(t, \xi)-\frac{\gamma}{2}-\frac{1}{2} \operatorname{div}_{\xi} b(t, \xi)\right)|X(t, \xi)|^{2}\right. \\
\left.+f(X(t, \xi)) X(t, \xi)-\frac{1}{2} \sum_{k=1}^{\infty} X^{2}(t, \xi) \mathrm{e}_{k}^{2}(\xi)\right) \mathrm{d} \xi+\eta \int_{\mathcal{O}_{0}} \mathrm{e}^{\gamma t}|X(t, \xi)|^{2} \mathrm{~d} \xi, \\
M(t)=\int_{0}^{t} \int_{\mathcal{O}} \sum_{k=1}^{\infty} \mathrm{e}^{\gamma s} X^{2}(s, \xi) e_{k}(s, \xi) \mathrm{d} \beta_{k}(s), t \geq 0 .
\end{gathered}
$$

By Lemma 3.1 and by (2.12), we see that, for $\eta \geq \eta_{0}$ sufficiently large and $0<\gamma \leq \gamma_{0}$ sufficiently small, we have

$$
K(t) \geq \varepsilon_{0} \int_{\mathcal{O}} \mathrm{e}^{\gamma t}|X(t, \xi)|^{2} \mathrm{~d} \xi, \forall t>0, \mathbb{P} \text {-a.s. }
$$

where $\varepsilon_{0}>0$. Taking expectation into (3.19), we obtain that

$$
\frac{1}{2} \mathrm{e}^{\gamma t} \mathbb{E}|X(t)|_{2}^{2}+\varepsilon_{0} \int_{0}^{t} \mathrm{e}^{\gamma s} \mathbb{E}|X(s)|_{2}^{2} \mathrm{~d} s \leq \frac{1}{2}|x|_{2}^{2}, \forall t \geq 0
$$

Since $t \rightarrow \int_{0}^{t} K(s)$ is an a.s. nondecreasing stochastic process, $\mathrm{e}^{\gamma t}|X(t)|_{2}^{2}$ is a nonnegative semi-martingale and $t \rightarrow M(t)$ is a continuous local martingale, we infer by Theorem 7 [13], page 139, that there exist $\mathbb{P}$-a.s.

$$
\lim _{t \rightarrow \infty}\left(\mathrm{e}^{\gamma t}|X(t)|_{2}^{2}\right)<\infty, K(\infty)<\infty,
$$

which, by virtue of (3.19), imply (2.13), (2.14), as claimed.

Remark 3.2. By the proof, it is clear that Theorem 2.1 extends to more general nonlinear functions $f=$ $f(t, \xi, x)$, as well as to smooth functions $\mu_{k}=\mu_{k}(t, \xi)$. Also, the Lipschitz condition (1.6) can be weakened to $f$ continuous, monotonically increasing and with polynomial growth. Moreover, $\Delta$ can be replaced by any strongly elliptic linear operator in $\mathcal{O}$. The details are omitted. 


\section{Stabilization of Navier-Stokes Equations WITH MULTIPLICATIVE NOISE}

We consider here the stochastic Navier-Stokes equation

$$
\begin{aligned}
& \mathrm{d} X(t)-\nu \Delta X(t) \mathrm{d} t+(a(t) \cdot \nabla) X(t) \mathrm{d} t+(X(t) \cdot \nabla) b(t) \mathrm{d} t+(X(t) \cdot \nabla) X(t) \mathrm{d} t \\
& \quad=X(t) \mathrm{d} W(t)+\nabla p(t) \mathrm{d} t+\mathbf{1}_{\mathcal{O}_{0}} u(t) \mathrm{d} t \quad \text { in } \quad(0, \infty) \times \mathcal{O} \\
& \nabla \cdot X(t)=0 \text { in }(0, \infty) \times \mathcal{O}, \\
& X(t)=0 \text { on }(0, \infty) \times \partial \mathcal{O}, X(0)=x \text { in } \mathcal{O},
\end{aligned}
$$

where $\nu>0, a, b \in\left(C^{1}((0, \infty) \times \overline{\mathcal{O}})\right)^{2}, \nabla \cdot a=\nabla \cdot b=0, a \cdot \boldsymbol{n}=b \cdot \boldsymbol{n}=0$ on $\partial \mathcal{O}$. Here $\mathcal{O}$ is a bounded and open domain of $\mathbb{R}^{2}$ and $\mathcal{O}_{0}$ is an open subset of $\mathcal{O}$. The boundaries $\partial \mathcal{O}$ and $\partial \mathcal{O}_{0}$ are assumed to be smooth. We set

$$
H=\left\{y \in\left(L^{2}(\mathcal{O})\right)^{2} ; \nabla \cdot y=0, y \cdot \boldsymbol{n}=0 \quad \text { on } \quad \partial \mathcal{O}\right\},
$$

where $\boldsymbol{n}$ is the normal to $\partial \mathcal{O}$. We denote by $\langle\cdot, \cdot\rangle_{H}$ the scalar product of $H$ and by $|\cdot|_{H}$ the norm. The Wiener process $W(t)$ is of the form $(1.2)$, where $\left\{e_{k}\right\} \subset\left(C^{2}(\overline{\mathcal{O}})\right)^{2}$ is an orthonormal basis in $H$, and $\mu_{k} \in \mathbb{R}$. As in the previous case, the main objective here is the design of a stabilizable feedback controller $u$ for equation (2.10).

We use the standard notations

$$
\begin{aligned}
& V=\left\{y \in H \cap\left(H_{0}^{1}(\mathcal{O})\right)^{2} ; \nabla \cdot y=0 \quad \text { in } \quad \mathcal{O}\right\}, \\
& A=-\nu \Pi \Delta, D(A)=\left(H^{2}(\mathcal{O})\right)^{2} \cap V,
\end{aligned}
$$

and rewrite (4.24) as

$$
\begin{aligned}
& \mathrm{d} X+A X \mathrm{~d} t+\Pi((a(t) \cdot \nabla) X+(X \cdot \nabla) b(t) \mathrm{d} t)=\Pi(X \mathrm{~d} W), \\
& X(0)=x,
\end{aligned}
$$

where $\Pi$ is the Leray projector on $H$. Consider the Stokes operator $A_{1}$ on $\mathcal{O}_{1}=\mathcal{O} \backslash \mathcal{O}_{0}$, that is,

$$
\left\langle A_{1} y, \varphi\right\rangle=\nu \sum_{i=1}^{2} \int_{\mathcal{O}_{1}} \nabla y_{i} \cdot \nabla \varphi_{i} \mathrm{~d} \xi, \forall \varphi \in V_{1}
$$

where $V_{1}=\left\{y \in\left(H_{0}^{1}\left(\mathcal{O}_{1}\right)\right)^{2} ; \nabla \cdot y=0\right.$ in $\left.\mathcal{O}_{1}\right\}$. Denote again by $\lambda_{1}^{*}\left(\mathcal{O}_{1}\right)$ the first eigenvalue of $A_{1}$, that is,

$$
\lambda_{1}^{*}\left(\mathcal{O}_{1}\right)=\inf \left\{\nu \sum_{i=1}^{2} \int_{\mathcal{O}}\left|\nabla \varphi_{i}\right|^{2} \mathrm{~d} \xi, \varphi=\left\{\varphi_{1}, \varphi_{2}\right\} \in V_{1}, \int_{\mathcal{O}_{1}}|\varphi|^{2} \mathrm{~d} \xi=1\right\} .
$$

Also, in this case, we have (see Lem. 1 in [4]), for $\eta \geq \eta_{0}(\varepsilon)$ and $\varepsilon>0$,

$$
\langle A y, y\rangle_{H}+\eta\left\langle\Pi\left(\mathbf{1}_{\mathcal{O}_{0}} y\right), y\right\rangle_{H} \geq\left(\lambda_{1}^{*}\left(\mathcal{O}_{1}\right)-\varepsilon\right)|y|_{H}^{2}, \forall y \in V .
$$

We consider in system (4.24) the linear feedback controller

$$
u=-\eta X, \eta>0 .
$$

We set

$$
\gamma^{*}(t)=\sup \left\{\int_{\mathcal{O}}\left|y_{i} D_{i} b_{j} y_{j} \mathrm{~d} \xi\right| ;|y|_{H}=1\right\}<\infty
$$


where $b=\left\{b_{1}, b_{2}\right\}$. We have

Theorem 4.1. Assume that

$$
\lambda_{1}^{*}\left(\mathcal{O}_{1}\right)>\frac{1}{2} \sum_{j=1}^{\infty} \mu_{j}^{2}\left|e_{j}\right|_{\infty}^{2}+\sup _{t \in \mathbb{R}^{+}} \gamma^{*}(t) .
$$

Then, for each $x \in H$ and $\eta$ sufficiently large and independent of $x$, the solution $X$ to the closed loop system (4.24) with the feedback controller (4.27) satisfies

$$
\begin{gathered}
\mathbb{E}\left[\mathrm{e}^{\gamma t}|X(t)|_{H}^{2}\right]+\int_{0}^{\infty} \mathrm{e}^{\gamma t} \mathbb{E}|X(t)|_{H}^{2} \mathrm{~d} t<C|x|_{H}^{2}, \\
\lim _{t \rightarrow \infty} \mathrm{e}^{\gamma t}|X(t)|_{H}^{2}=0, \mathbb{P} \text {-a.s. },
\end{gathered}
$$

for some $\gamma>0$.

The proof is essentially the same as that of Theorem 2.1, and so it will be sketched only. Taking into account that

$$
\langle(X \cdot \nabla) X, X\rangle_{H}+\langle(a(t) \cdot \nabla) X, X\rangle_{H}=0, t>0, \mathbb{P} \text {-a.s., }
$$

we obtain by $(4.24),(4.27)$, via Itô's formula, that

$$
\begin{gathered}
\frac{1}{2} \mathrm{e}^{\gamma t}|X(t)|_{H}^{2}+\int_{0}^{t} \mathrm{e}^{\gamma s}\left(\langle A X(s), X(s)\rangle_{H}+\langle X(s) \cdot \nabla b(s), X(s)\rangle_{H}\right. \\
\left.-\frac{\gamma}{2}|X(s)|_{2}^{2}-\frac{1}{2} \sum_{j=1}^{\infty}\left|X(s) e_{j}\right|_{H}^{2}+\eta\left\langle\mathbf{1}_{\mathcal{O}_{0}} X(s), X(s)\right\rangle_{H}\right) \mathrm{d} s \\
=\frac{1}{2}|x|_{H}^{2}+\int_{0}^{t} \mathrm{e}^{\gamma s} \sum_{j=1}^{\infty}\left\langle X(s) e_{j}, X(s)\right\rangle_{H} \mathrm{~d} \beta_{j}(s), t \geq 0 .
\end{gathered}
$$

Then, by virtue of (4.26) and (4.28), we have, by (4.31), that

$$
\frac{1}{2} \mathrm{e}^{\gamma t}|X(t)|_{H}^{2}+I(t)=\frac{1}{2}|x|_{H}^{2}+M^{*}(t), t \geq 0, \mathbb{P} \text {-a.s. }
$$

where the first left hand side term is a nonnegative semi-martingale, $I(t)$ is a nondecreasing process, which satisfies

$$
\mathbb{E}[I(t)] \geq \varepsilon_{0} \int_{0}^{t} \mathrm{e}^{\gamma s} \mathbb{E}|X(s)|_{H}^{2} \mathrm{~d} s, \forall t \geq 0,
$$

for $\eta$ sufficiently large, and $M^{*}(t)=\int_{0}^{t} \mathrm{e}^{\gamma s} \sum_{j=1}^{\infty}\left\langle X(s) e_{j}, X(s)\right\rangle_{H} \mathrm{~d} \beta_{j}(s)$ is a continuous local martingale. As in the previous case, this implies via Theorem 7 [13] that $\lim _{t \rightarrow \infty} \mathrm{e}^{\gamma t}|X(t)|_{H}^{2}$ exists $\mathbb{P}$-a.s. and, therefore, (4.29) and (4.30) hold.

\section{FINAL REMARKS}

In order to make clear the novelty of the above results and the principal difficulties related to the internal stabilization of equations (1.1) and (4.24), we note that, via the substitution $y=\mathrm{e}^{W(t)} X$, equation (1.1) reduces to a parabolic equation of the form

$$
\begin{aligned}
& \frac{\partial y}{\partial t}-\Delta y+\widetilde{a}(t) y+\widetilde{b}(t) \cdot \nabla y+\frac{1}{2} \sum_{i=1}^{\infty} \mu_{k}^{2} \mathrm{e}_{k}^{2} y=\mathbf{1}_{\mathcal{O}_{0}} u, \mathbb{P} \text {-a.s., } \\
& y=0 \quad \text { on } \quad(0, \infty) \times \partial \mathcal{O},
\end{aligned}
$$

with random coefficients $\widetilde{a}, \widetilde{b}$. 
If $\widetilde{a}$ and $\widetilde{b}$ are independent functions of $t$, then (5.32) can be stabilized by a controller $u=\sum_{j=1}^{N} u_{j}(t) \psi_{j}$, where $\psi_{j}$ are linear combinations of eigenfunctions of the dual operator $y \rightarrow-\Delta y+\widetilde{a} y-\operatorname{div}(b y)$ (see [7] or [3] for the case of Navier-Stokes equations).

For deterministic equations with smooth time dependent coefficients, a similar result was recently proved in [6] (for the Navier-Stokes equations), but it cannot be applied, however, to the random equation (5.32) since it does not provide an adapted stabilizable controller $u=u(t)$. (The reason is that the argument in [6] relies on exact $\mathbb{P}$-a.s. controllability of (5.32) via an adapted controller $u$ which so far is still an open problem).

By the new procedure we use here, we circumvert this basic difficulty by constructing an explicit feedback adapted controller and avoiding so the exact controllability of the stochastic equation with multiplicative noise which is equivalent to an open problem: observability inequality for the dual stochastic equation.

On these lines, we briefly present below a partial result on exact controllability of the linear Stokes equation (4.24), that is,

$$
\begin{aligned}
& \mathrm{d} X-\nu \Delta X \mathrm{~d} t+(a \cdot \nabla) X \mathrm{~d} t+(X \cdot \nabla) b \mathrm{~d} t=X \mathrm{~d} W+\nabla p \mathrm{~d} t+\mathbf{1}_{\mathcal{O}_{0}} u \mathrm{~d} t \\
& X=0 \quad \text { on } \quad(0, \infty) \times \partial \mathcal{O}, \quad X(0)=x \quad \text { in } \mathcal{O},
\end{aligned}
$$

which can be obtained by such a rescaling argument in the special case, where

$$
W(t)=\sum_{j=1}^{M} \mu_{j}(t) \beta_{j}(t),
$$

where $\mu_{j}$ are adapted processes which are in $L^{\infty}((0, T) \times \Omega)$. By the transformation $X=\exp \left(\int_{0}^{t} \sum_{j=1}^{M} \mu_{j} \mathrm{~d} \beta_{j}\right) y$, we reduce equation (5.33) via Itô's formula to

$$
\begin{aligned}
& \frac{\partial y}{\partial t}-\nu \Delta y+(a(t) \cdot \nabla) y+(y \cdot \nabla) b(t)+\widetilde{\mu}(t) y=\mathbf{1}_{\mathcal{O}_{0}} v \quad \text { in } \quad(0, T) \times \mathcal{O}, \\
& y(0)=x \quad \text { in } \quad \mathcal{O}, \\
& \nabla \cdot y=0, y=0 \quad \text { on } \quad(0, T) \times \partial \mathcal{O} .
\end{aligned}
$$

Here, $v(t)=\exp \left(-\int_{0}^{t} \sum_{j=1}^{M} \mu_{j} \mathrm{~d} \beta_{j}\right) u, \widetilde{\mu}=\frac{1}{2} \sum_{j=1}^{M} \mu_{j}^{2}$.

We set $z(t)=\exp \left(\int_{0}^{t} \widetilde{\mu}(s) \mathrm{d} s\right) y(t)$. Then, (5.35) reduces to

$$
\begin{aligned}
& \frac{\partial}{\partial t} z-\nu \Delta z+(a(t) \cdot \nabla) z+(z \cdot \nabla) b(t)=\mathbf{1}_{\mathcal{O}_{0}} \exp \left(\int_{0}^{t} \widetilde{\mu}(s) \mathrm{d} s-\int_{0}^{t} \sum_{j=1}^{M} \mu_{j}(s) \mathrm{d} \beta_{j}(s)\right) u=\mathbf{1}_{\mathcal{O}_{0}} v(t), \\
& z(0)=x \quad \text { in } \mathcal{O}, \\
& \nabla \cdot z=0, z=0 \quad \text { on } \quad(0, T) \times \partial \mathcal{O} .
\end{aligned}
$$

On the other hand, we know (see [14]) that (5.36) is exactly null controllable, that is, there is a controller $v \in L^{2}(0, T) \times \mathcal{O}$ such that $z(T) \equiv 0$. This means that $(5.35)$ is exactly null controllable by the adapted (progressively measurable) controller

$$
u(t, \xi)=\exp \left(-\int_{0}^{t} \sum_{j=1}^{M} \mu_{j} \mathrm{~d} \beta_{j}+\int_{0}^{t} \tilde{\mu} \mathrm{d} s\right) v(t, \xi) .
$$

We have proved, therefore,

Theorem 5.1. There is an adapted controller $u$ such that the solution $X$ to (5.33) satisfies $X(T, \xi) \equiv$ $0, \mathbb{P}$-a.s., $\xi \in \mathcal{O}$. 
Remark 5.2. A similar result can be obtained via the above argument on the parabolic equation (1.1) in the special case where the Gaussian process $W$ is of the form (5.34) with $\left\{\mathcal{F}_{t}\right\}$-adapted coefficients $\mu_{j}$. Such a result was obtained in [9] by a more intricate argument involving the Carleman inequality for the stochastic backward equation. It should be said, however, that the results of [9] refer to a controller $\mathbf{1}_{\mathcal{O}_{0}} \mathbf{1}_{\mathbb{E}} u$, where $\mathbb{E}$ is a measurable set of $(0, T)$.

\section{REFERENCES}

[1] S. Aniţa, Internal stabilization of diffusion equation. Nonlinear Stud. 8 (2001) 193-202.

[2] V. Barbu, Controllability of parabolic and Navier-Stokes equations. Sci. Math. Japon. 56 (2002) 143-211.

[3] V. Barbu, Stabilization of Navier-Stokes Flows, Communication and Control Engineering. Springer, London (2011).

[4] V. Barbu and C. Lefter, Internal stabilizability of the Navier-Stokes equations. Syst. Control Lett. 48 (2003) $161-167$.

[5] V. Barbu, A. Rascanu and G. Tessitore, Carleman estimates and controllability of linear stochastic heat equations. Appl. Math. Optimiz. 47 (2003) 1197-1209.

[6] V. Barbu, S.S. Rodriguez and A. Shirikyan, Internal exponential stabilization to a nonstationary solution for 3-D Navier-Stokes equations. SIAM J. Control Optim. 49 (2011) 1454-1478.

[7] V. Barbu and R. Triggiani, Internal stabilization of Navier-Stokes equations with finite-dimensional controllers. Indiana Univ. Math. J. 53 (2004) 1443-1494.

[8] G. Da Prato and J. Zabczyk, Ergodicity for Infinite Dimensional Systems. Cambridge University Press, Cambridge (1996).

[9] Qi, Lü, Some results on the controllability of forward stochastic heat equations with control on the drift. J. Funct. Anal. 260 (2011) 832-851.

[10] G. Da Prato and J. Zabczyk, Stochastic Equations in Infinite Dimensions. Cambridge University Press, Cambridge (2008).

[11] D. Goreac, Approximate controllability for linear stochastic differential equations in infinite dimensions. Appl. Math. Optim. 53 (2009) 105-132.

[12] O. Imanuvilov, On exact controllability of the Navier-Stokes equations. ESAIM: COCV 3 (1998) 97-131.

[13] R.S. Lipster and A. Shiryaev, Theory of Martingales. Kluwer Academic, Dordrecht (1989).

[14] S. Tang and X. Zhang, Null controllability for forward and backward stochastic parabolic equations. SIAM J. Control Optim. 48 (2009) 2191-2216 